\title{
Developmental changes in the influence of conventional and instrumental cues on over-imitation in 3- to 6-year-old children
}

\author{
Cristina-Andreea Moraru ${ }^{a}$, Juan-Carlos Gomez a , Nicola McGuigan ${ }^{\mathrm{b},{ }_{\mathrm{a}}}$ \\ School of Psychology and Neuroscience, St Andrews University, St Andrews, KY16 9JP, UK \\ ${ }^{\mathrm{b}}$ Department of Psychology, School of Life Sciences, Heriot Watt University, Edinburgh EH14 4AS, UK
}

Journal of Experimental Child Psychology 145 (2016) 34-47

\begin{abstract}
Previous studies have shown that children in the preschool period are fastidious imitators who copy models with such high levels of fidelity that task efficiency may be compromised. This over- imitative tendency, and the pervasive nature of it, has led to many explorations and theoretical interpretations of this behavior, including social, causal, and conventional explanations. In support of the conventional account, recent research has shown that chil- dren are more likely to overimitate when the task is framed using conventional verbal cues than when it is framed using instrumen- tal verbal cues. The aim of the current study was to determine whether 3- to 6-year-old children $(\mathrm{N}=185$, mean age $=60$ months $)$ would overimitate when presented with instrumental and con- ventional verbal cues, which varied only minimally and were more directly comparable between instrumental and conventional con- texts than those used in previous studies. In addition to varying the overall context, we also varied the instrumental prompt used such that the cues provided ranged in the extent to which they pro- vided explicit instruction to omit the irrelevant actions. Counter to our predictions, and the high levels of over-imitation witnessed in previous studies, the older children frequently over-imitated irre- spective of the context provided, whereas the youngest children over-imitated selectively, including the irrelevant actions only when the task was presented in a conventional frame. We propose that the age differences found following an instrumental
\end{abstract}

\section{Introduction}

Since the seminal research of Piaget a whole plethora of studies have shown that children are fastidious imitators who copy the actions performed by adults with extremely high levels of fidelity (e.g., Hopper, Lambeth, Schapiro, \& Whiten, 2008; McGuigan, 2014; Nagell, Olguin, \& Tomasello, 1993; Whiten, Custance, Gomez, Teixidor, \& Bard, 1996; Whiten \& Flynn, 2010). This approach to learning would appear highly adaptive as it prevents the observer having to learn new behaviors from scratch, a trial and error process which is potentially time consuming and error prone. However, recent studies have shown 
that this tendency towards high fidelity imitation, although generally useful, can sometimes lead children to act with reduced levels of efficiency. The now classic experimental demonstration of this reduction in efficiency was provided by Horner and Whiten (2005). In this study young children watched whilst an adult demonstrator extracted a reward from inside a puzzle box using a sequence of actions which included both causally necessary and causally unnecessary elements. Surprisingly, rather than omitting the irrelevant actions the children copied all elements of the display with extreme precision. This over-imitative tendency has since been replicated in both Western and Non-Western cultures (Berl \& Hewlett, 2015; Nielsen, Mushin, Tomaselli \& Whiten, 2014; Nielsen \& Tomaselli, 2010) using a variety of different tasks containing the same basic principles as Horner and Whiten's original study. The consistent pattern which emerges from these studies is one in which overimitation increases with age, continually rising from the preschool period through to adulthood (McGuigan, Whiten, Flynn, \& Horner, 2007; McGuigan, Gladstone \& Cook, 2012; McGuigan, Makinson, \& Whiten, 2011).

The curious nature of this over-imitative response has generated many different theoretical interpretations. The 'Automatic Coding Hypothesis' and the related 'Copy-allrefine-later hypothesis', suggest that over-imitation occurs as a result of children encoding all of the intentional actions performed by an adult as causally meaningful (Lyons, Damrosch, Lin, Macris, \& Keil, 2011; Lyons, Young, \& Keil, 2007; Whiten, McGuigan MarshallPescini, Hopper, 2009). Under this account the children experience causal confusion, mistakenly believing that the irrelevant actions are causally necessary for successful task performance. This hypothesis is supported by the pervasiveness of over-imitation across studies, even under conditions which have attempted to reduce the occurrence of overimitation, for example by training children to identify 'silly' actions (Lyon et al., 2007), or by allowing children to attempt the task before viewing an inefficient demonstration (Nielsen \& 
Tomaselli, 2010). In contrast the 'Social Affiliation Hypothesis' views over-imitation as an active attempt to affiliate with, or 'to be like', the model (Nielsen, 2006). Under this account the participants are aware that the actions are causally unnecessary, and are only reproducing these actions for social reasons. The social affiliation account is supported by the finding that children copy irrelevant actions selectively, including them in the presence of the inefficient demonstrator that used them, but not in the presence of the an efficient demonstrator that used causal actions only (Nielsen \& Blank, 2011). A third theoretical account of overimitation proposes that children include causally irrelevant actions in their reproductions due to their acquisition of a prescriptive norm of how particular objects should be operated (Kenward, Karlsson, \& Persson, 2011; Kenward, 2012). In this case the children view the irrelevant actions as conventionally, rather than, causally necessary, an account which is supported by the finding that children who have previously seen an inefficient adult model include irrelevancies in their demonstration frequently protest against puppets who subsequently omit the irrelevant actions from their reproduction (Keupp, Behne, \& Rakoczy, 2013).

In an attempt to tease apart these different explanations for high fidelity copying recent studies have manipulated the extent to which conventionality, or instrumentality, is displayed within the experimental context (see Legare \& Nielsen, in press for a review). Instrumental actions are causally transparent, and the acquisition of such actions allows the observer to learn the key skills required in their environment (e.g., how to use tools). In contrast, social conventions (e.g., the way in which we eat or dress) are defined as "causally opaque, socially shared actions" (Watson-Jones, Legare, Whitehouse \& Clegg, 2014), which are believed to serve an important role in enhancing affiliation and co-operation, and avoiding ostracism, within social groups. In order that children learn both instrumental and conventional actions imitation must be multifunctional, allowing children to selectively copy 
in a variety of different situations. This task is necessarily complex, requiring children to use a range of social and contextual cues to determine the goal of a particular behavior, and subsequently adopt the appropriate approach for the specific context.

The complexity facing the observer is illustrated by the number of different ways that conventionality can be displayed in the social learning context. Cues which indicate conventionality highlight that this is 'how' an action should be performed, and can be manipulated by increasing the number and synchronicity of the models (Herrmann, Legare, Harris, \& Whitehouse, 2013), or by highlighting the causal opacity of the actions, either by having an identical start- and end-state to the task (Legare, Wen, Herrmann \& Whitehouse, 2015; Watson-Jones et al, 2014), or by performing the task outcome before performing the irrelevant actions (Nielsen, Kapitány \& Elkins, 2015). An alternative way to manipulate conventionality is to vary the overall communicative context in which the task is presented. Such contextual variations include changes to the social context, for example by altering the amount of pedagogical cues (e.g., looking or not looking at the child during the task demonstration) provided by the models (Brugger, Lariviere, Mumme, \& Bushnell, 2007; Buchsbaum, Gopnik, Griffiths, \& Shafto, 2011; Hoehl, Zettersten, Schleihauf, Grätz, \& Pauen, 2014), or through establishing different contexts by allowing children to play either a 'copy-me-game', or an instrumental 'shared goal' game, with the model (Yu \& Kushnir, 2014). Despite differences in the way in which conventionality was displayed across these studies the majority have shown increases in imitative fidelity with increases in conventionality, with both greater imitative fidelity, and greater discrimination occurring between contexts as children age. The exception to this pattern came in $\mathrm{Yu}$ and Kushnir (2014) where the older children discriminated less between the instrumental and conventional contexts than their younger counterparts, suggesting that the interaction between observer age and the social context in which the task is presented is complex. 
An alternative to using social context as a mechanism to establish the task framework is to use verbal cues. Herrmann et al. (2013) presented 3- to 6-year-old children with a video in which one or more models used a particular sequence of actions to remove pegs from a pegboard. The task demonstration was either framed in a conventional way ("She always does it this way"), or was framed as outcome oriented ("She gets pegs up"). In the conventional condition the children copied the model's actions with high levels of fidelity, and provided conventional justifications for their response ("I had to do it the way they did $\left.i t^{\prime \prime}\right)$. In contrast, when the task was framed as outcome-oriented, the children were less likely to copy precisely, and provided instrumental justifications that emphasised independent agency ("I can do whatever I want"). These findings have recently been shown to extend to a more naturalistic context in which children asked to make a necklace were more likely to copy irrelevant elements of the display (e.g., touch a bead on their forehead before placing it on a string) with higher levels of fidelity when the task was labelled conventionally ("Everyone does it this way"), rather than instrumentally ("I'm going to make a necklace”) (Clegg \& Legare, in press). Importantly, recent research has shown that for high fidelity matching to occur the context in which the task is presented must match the subsequent task instructions, with actions initially labelled as conventional (e.g., described as "daxing”) being reproduced less faithfully following post-demonstration instrumental instructions ("have a go and find a puzzle piece"), than post-demonstration conventional instructions (“have a go and dax”) (Keupp, Behne, Zachow, Kasbohm, \& Rakoczy, 2015).

Taken together the research outlined above suggests that young children are extremely sensitive to the conventional or instrumental context in which the social learning experience is framed, and alter their copying behavior accordingly. However, despite displaying such context sensitivity, other recent studies have shown that the context in which a task was presented had little influence on the levels of over-imitation witnessed (Keupp et 
al., 2013). Keupp et al. (2013) presented 3- and 5-year-old children with a task demonstration in which the model performed both causally irrelevant actions and causally relevant actions before an effect was produced (e.g., ringing a bell). The task was presented in one of two contexts, either instrumental or conventional. In the conventional context conventionality was established in two ways, first by allowing the children to view the effect produced (using only goal relevant actions) prior to the task demonstration, and second by providing the irrelevant action with a novel label (e.g., “now I'm going to dax”). The instrumental context contained no prior demonstration of the effect, and the action label used by the model referred directly to the effect ("now I'm going to ring the bells"). The results showed that the levels of over-imitation were high, and did not vary according to the context in which the task was presented. However, children in the conventional condition were more likely to protest against a puppet who subsequently omitted the causally irrelevant actions than those in the instrumental condition suggesting that the children were aware of the conventionality of the actions. The variation in the imitative fidelity in these studies suggests that the impact of establishing conventionality and instrumentality on over-imitation is complex, and further research is needed to establish the conditions under which children will display selective copying in normative and instrumental contexts..

The primary aim of the current study was to explore the influence of conventional and instrumental verbal framing on over-imitation using a more minimal change in the verbal cues used to establish each context than included in the studies described above. Previous studies have typically established an instrumental context by explicitly focusing the attention of the observer towards material aspects of the task (e.g., pegs), whereas the conventional context is often established by reference to non-material aspects (e.g., the usual way actions are performed). Such variation in where the attention of the observer was directed may have led to the contextual differences witnessed as a result of more superficial attentional 
processes than to the different contextual cues per se. In order to rule out the influence of these more basic attentional factors we held reference to the non-material aspects of the task constant across contexts using a very minimal change in the pre-demonstration language used in order to indicate that there was a single conventional solution ("I will show you how to get the toy out"), or multiple (instrumental) solutions ("I will show you one way to get the toy out"). Varying the context in this way allowed us to determine whether children's selective adoption of either a conventional, or an instrumental, stance is so powerful that it could be elicited from even minimal verbal cues.

Our second aim was to provide the first exploration of the influence that different post-demonstration instrumental instructions have on copying fidelity. In order to achieve this we varied the post-demonstration instructions in the multiple solutions condition such that they differed in the extent to which they explicitly indicated that children could omit the irrelevant actions from their reproduction. This ranged from little explicit instruction ( 'now it's your turn'), to ('you can do it however you want'), to highly explicit instruction ('avoid silly actions '). The different instrumental prompts were included in order that we could explore how readily children use the verbal cues provided by the demonstrator in order to approach the task instrumentally. It may be the case that an instrumental stance is most strongly elicited following a task instruction which explicitly indicates that the irrelevant actions contained in the demonstration are unnecessary. Alternatively the presence of less explicit cues which indicate the existence of other task solutions may be enough to trigger an instrumental stance. By providing a range of such cues we aimed to provide the most detailed exploration to date of children's sensitivity to verbal instrumental cues. These postdemonstration instructions were not provided in the single solution condition due to a potential conflict with the conventional nature of the pre-demonstration prompt. We predicted that under a normative/conventional account the level of over-imitation would be 
higher following a pre-demonstration prompt that referred to a single conventional solution as opposed to multiple solutions. In contrast, if children were over-imitating for either social affiliative reasons, or due to causal confusion, then the levels of over-imitation would be predicted to be high irrespective of the context in which the task was presented. With respect to the post-demonstration instructions in the multiple solutions conditions, we predicted that the level of over-imitation would vary according to how directly the instruction intimated that the irrelevant actions could be omitted, with the smallest reduction in over-imitation occurring in the 'your turn' condition and the largest reduction occurring in the 'avoid silly actions' condition.

\section{Method}

Participants

A total of 185 children participated in the study. The children were recruited from four different age groups: a 3-year-old group (20 males, 21 females; mean age $=43$ months, range 36-47 months, $\mathrm{SD}=3$ months), a 4-year-old group (20 males, 23 females; mean age $=54$ months, range 48-59 months, $\mathrm{SD}=4$ months), a 5-year-old group (33 males, 24 females; mean age $=65$ months, range $60-71$ months, $\mathrm{SD}=4$ months $)$ and a 6 -year-old group (22 males, 22 females; mean age $=77$ months, range 72-83 months, $\mathrm{SD}=4$ months). Participants were recruited at the Budongo Trail Centre, a public engagement facility at Edinburgh Zoo, UK. As a consequence of the environment in which the task was presented an additional 65 children were excluded from the study due to parental intervention, an inability to attend to the task, a refusal to engage with the task or experimenter error.

\section{Apparatus}

The apparatus consisted of a puzzle-box initially used by Horner and Whiten (2005) to 
explore over-imitation in children within the preschool period. The box was made of transparent polycarbonate $\left(20 \mathrm{~cm}^{3}\right)$, which was completely enclosed with the exception of two holes $(2 \mathrm{~cm} \mathrm{x} 2 \mathrm{~cm})$, one located on top of the box and one located on the front face of the box. Each of the holes was covered by a defence which had to be removed in order to allow access to the hole. The defence covering the top hole comprised two bolts which had to be pushed to one side to allow access to the hole beneath. The defence covering the lower hole was a small door which had to be slid to one side in order to allow access to the hole behind. The lower hole was connected to an opaque tube that housed a hidden reward (a toy with a magnet attached). In order to retrieve the reward participants only had to perform two causally relevant actions: 1) slide open the door defence, and 2) insert a magnetic tipped tool $(22 \mathrm{~cm}$ long) into the tube behind. In contrast, removing the bolt defence and inserting the tool into the top hole only resulted in the tool hitting a transparent barrier that prevented the tool from touching the opaque tube. Therefore, tool use towards the box could be classified as either causally relevant (directed to the front of the box), or causally irrelevant (directed to the top of the box).

\section{Design}

Participants were allocated to one of four conditions using a between participants design. The conditions varied according to the content of the verbal instructions given before and after the task demonstration. In one condition the pre-demonstration instructions indicated that there was only one way to obtain the reward (single solution-conventional stance; see below for exact wording), whereas the instructions for the remaining three conditions indicated that there was more than one way to retrieve the reward from the box (multiple solutionsinstrumental stance). The post-demonstration instruction provided in the three multiple solutions conditions was varied to provide increasingly more direct prompts to omit the 
irrelevant actions (see below for exact wording of the conditions).

\section{Procedure}

Children were recruited through posters displayed in the Budongo Trail facility at Edinburgh Zoo, UK. The children were tested individually in a quiet area of the facility which was enclosed such that children who were waiting to participate could not see the experiment taking place. Upon entering the testing area each child was asked to sit at a small table, with the female experimenter positioned directly opposite. Once the child appeared comfortable the experimenter revealed the box (which was previously hidden out of view) and provided the child with either: 1) the 'single solutions' prompt: 'This is my box. There is a toy inside it. Now, I want you to look closely, because I will show you how to get the toy out', or 2) the 'multiple solutions prompt': 'This is my box. There is a toy inside it. Now, I want you to look closely, because I will show you one way to get the toy out'. After the child received the appropriate prompt, the experimenter provided a task demonstration containing five causally irrelevant actions (both bolts were removed in order to uncover the top hole and the tool was inserted into the top hole three times) followed by three causally relevant actions (the door was slid open, the tool inserted into the hole behind, and the reward extracted).

Once the experimenter had successfully retrieved the reward the children were asked to close their eyes while the box was reset. Upon reconfiguration of the box the children were asked to open their eyes, and the second part of the task instructions was given. The specific instruction given to the participants was varied such that the omission of the irrelevant actions in the multiple solutions conditions (one way to get the toy') became increasingly explicit with the following post-demonstration instructions: 1) 'Now it's is your turn' (Multiple solutions-Your Turn), 2) 'Now it's your turn. You can get the toy out however you 
want' (Multiple Solutions-However) or 3) 'I want you to know that some of the things I did when I got the toy out were silly. I did not need to do them to get the toy out. So I want you to try your best and not do those things. Now it is your turn' (Multiple Solutions-Silly). The least explicit instruction was provided in the 'Single Solution-Your Turn' condition in which the earlier 'how to get the toy out' was followed with by 'Now it's your turn'.

On completion of the task instructions the box was passed to the participant who was allowed to attempt the task until: 1) they had successfully retrieved the reward from the box, 2) 3 minutes had elapsed, or 3) they withdrew from the task. The trials of all participants were video-taped for further analysis. On completion of the experiment all children received a sticker as a gift for taking part irrespective of whether or not they successfully retrieved the reward.

\section{Scoring}

An over-imitation score was calculated for each participant by totalling the number of irrelevant actions performed. A score of 0 was allocated to participants who performed no irrelevant actions, with a score of 5 being given for reproduction of all of the irrelevant components. The order in which the irrelevant actions were performed was not taken into consideration in the scoring as little variation in the order of reproduction was possible due to the bolts having to be removed before the irrelevant tool insertions could be performed. Similarly, a relevant action score was calculated by totalling the number of goal relevant actions performed by each participant. This score could range from $0-3$, and as with the irrelevant actions order was not taken into account due to the necessity of having to perform the actions in a set sequence (i.e., the door had to be opened before the tool could be inserted to retrieve the reward). 


\section{Inter-rater reliability}

The data from nineteen children, representing $10 \%$ of the total sample, were re-coded by an independent observer, who was naive to the hypothesis of the experiment. There was $100 \%$ agreement across raters for both irrelevant and relevant actions.

\section{Results}

Successful toy retrieval

Of the 185 children who took part in the experiment 167 successfully retrieved the reward from the box. The remaining 18 children inserted the tool in the lower hole but failed to retrieve the reward either because they used the non-magnetic end of the tool, or because the reward got stuck in the tube. The over-imitation score of these 18 individuals comprised the total number of irrelevant actions performed up to the point the child would have retrieved the reward (i.e., any irrelevant actions performed after a failed retrieval attempt were not included).

\section{Pre-demonstration prompt: Single versus multiple solutions}

Of initial interest was whether the participants in the 'Single Solution' condition would perform a greater number of causally irrelevant actions than the participants in the 'Multiple Solutions' conditions, and whether this would vary according to the gender and the age of the participants. The participants over-imitation scores were analysed using a univariate GLM with condition (multiple or single) and participant gender (male or female) as the between participants factors, and age as a continuous variable. The analysis revealed a significant main effect of condition with the children performing significantly more causally irrelevant actions in the 'Single Solutions' condition (mean = 2.5) than the 'Multiple Solutions' 
conditions $\left(\right.$ mean $=2.1 ; \mathrm{F}(1,179)=5.4, \mathrm{p}=.02$, partial $\eta^{2}=.03$; see Fig. 1$)$. The analysis also revealed a marginally significant main effect of age $\left(F(1,179)=3.2, p=.07\right.$, partial $\eta^{2}=$ $.02)$, as well as a significant interaction between age and condition $(\mathrm{F}(1,179)=4.3, \mathrm{p}=.04$, partial $\eta^{2}=.02$ ) with over-imitation increasing with increasing age in the 'Multiple Solutions' conditions, and varying little with age in the 'Single Solutions' condition (see Fig. 1). See Table 1 for a complete breakdown of performance by age group and condition. No gender differences were revealed in the analysis.

\section{Post-demonstration prompt: Increasing permissiveness}

As well as determining whether there were differences in over-imitation following multiple and single solution prompts pre-demonstration we were also interested in whether the specific post-demonstration prompt provided in the 'Multiple Solutions' conditions influenced the tendency of children to include the irrelevant actions. In order to explore this possibility the participants over-imitation scores were analysed using a univariate GLM with condition (Multiple Solutions-Your Turn; Multiple Solutions-However; Multiple SolutionsSilly) and participant gender (male or female) as the between participants factors, and age as a continuous variable. The analysis revealed a significant main effect of condition $(F(2,131)$ $=3.4, \mathrm{p}=.04$, partial $\left.\eta^{2}=.05\right)$, and age $\left(\mathrm{F}(1,131)=15.4, \mathrm{p}<.001\right.$, partial $\left.\eta^{2}=.11\right)$, as well as a significant interaction between age and condition $\left(\mathrm{F}(2,131)=4.1, \mathrm{p}=.02\right.$, partial $\left.\eta^{2}=.06\right)$. Over-imitation was highest (mean $=2.4$ ), and increased with age, in the 'Multiple SolutionsYour Turn' condition, whereas in the 'Multiple Solutions- Silly' condition the rate of overimitation was somewhat lower $($ mean $=1.9)$, and remained flat across the entire age period (see Fig. 2). The influence of observer age was also apparent in the 'Multiple SolutionsHowever' condition (mean $=2.0$ ) where increasing age led to an increase in the level of overimitation witnessed (See Fig. 2). Follow up post-hoc Tukey tests revealed that the number of 
irrelevant actions performed in the 'Multiple Solutions-Your Turn' condition were marginally greater than those performed in the 'Multiple Solutions-Silly' condition $(p=.08)$, whereas the remaining condition comparisons failed to approach significance. See Table 1 for a complete breakdown of performance by age group and condition. No gender differences were revealed in the analysis.

\section{Discussion}

The aim of the current study was to use a puzzle-box task which has previously been shown to elicit high levels of over-imitation in preschool children in order to determine: 1) whether making very minimal changes to the verbal framework (instrumental or conventional) provided prior to the task demonstration, whilst keeping reference to the non-material aspects of the task constant across contexts, would influence the tendency of children to over-imitate, 2) whether varying the post-demonstration instruction to include differing degrees of direct instruction to omit the irrelevant actions would influence the degree of over-imitation witnessed, and 3) to determine whether there were age differences in how the children responded to both the contextual variation established pre-demonstration, and the level of permissiveness established post-demonstration. The results showed that in the conventionally framed 'Single-Solution' condition children across the age range copied the irrelevant actions with high levels of fidelity. However, performance was more variable when the task was instrumentally framed in the 'Multiple Solutions' condition; whereas the oldest children most often over-imitated as with the conventional framing, the level of over-imitation found in the youngest children fell substantially when the possibility of multiple solutions was prompted before demonstration. With respect to the post-demonstration instructions in the multiple solutions conditions highlighting to different degrees the existence of "silly", unnecessary actions, only the oldest children showed any sensitivity between conditions, performing 
fewest irrelevant actions when asked to omit 'silly' actions. Thus consistent with the greater imitative fidelity, and context discrimination, witnessed in previous studies (e.g., Herrmann et al., 2013) it appears that children across the age span tested can act on very subtle differences in the task instructions provided in order to imitate, or copy more selectively, but the way in which they do so varies with age.

That the older children displayed less context sensitivity than the younger children, following the pre-demonstration prompt in the majority of conditions may be explained by taking into account the motivation behind the behavior, in particular the specific goal that the observer holds in relation to the task. Over and Carpenter (2012) suggested that in a social learning situation the observer's goal could be to learn something new, to engage socially with the model, or a combination of the two. In the case where a learning goal predominates the child is motivated to learn a new skill, and as a result will focus on the elements of the task that they deem important for success. Social relations with the model are not as important when the child is motivated to learn, hence the causality of the task can outweigh the technique demonstrated. In contrast, where social goals predominate, learning a new skill is not of paramount importance; instead the goal is to build a relationship with, or be like, the model. In this case the model's behavior is the main focus and one would expect the child to engage in high fidelity copying. In the context of the current task we may expect that such differences in motivation would lead the younger children, who are less cognitively mature and lacking in experience, to be motivated to learn how the task works (and consequently focus on the causality of the task and omit the irrelevant actions), and the older children, who more readily understand the causality of the task, to be more socially motivated and consequently over-imitate.

The results of the current study are on the one hand supportive of the suggestion that different learning motivations predominate at different ages, however our findings 
indicate that the developmental picture is more complex, with different underlying motivations interacting with the framework indicated by the task instructions. The youngest children in our study, 3-year-olds, tended to find their own more efficient solution to the task unless it was directly intimated to them that the inefficient way was 'how' the task should be solved. The faithful reproduction following the conventional 'how' instruction suggests that the youngest children were sensitive to the normative nature of the single solution instruction, i.e., this is the correct way to operate this object. This interpretation fits well with the results of previous studies which have found that young children, even from the age of two years, are sensitive to normativity, making normative protests towards individuals who perform a task in an unconventional way in both the context of social learning (Kenward, 2012; Keupp et al., 2013; Keupp et al., 2015), and game play (Rakoczy, Brosche, Warneken, \& Tomasello, 2009; Rakoczy, Warneken, \& Tomasello, 2008, 2009). That our 3-year-olds failed to reproduce the causally irrelevant actions in the conditions where the possibility of multiple solutions was highlighted before the demonstration suggests that these children were neither causally confused, nor engaging in social affiliation per se. Instead, they appeared to use the more open instruction as a cue to operate the task instrumentally and learn the causality of the object. The ability of 3-year-old children to operate efficiently despite having viewed a single inefficient adult model is counter to previous over-imitation studies, where the standard predemonstration instruction 'watch what happens' followed by a 'your turn' instruction typically results in high levels of over-imitation in this age group (e.g., McGuigan et al., 2007). Taken together these findings suggest that if neither a conventional stance, or an instrumental stance, is directly intimated to the child, they view the demonstration as normative and include all the model's actions irrespective of causal relevance. However, this is not an absolute bias, as they show the flexibility of adapting their imitative response to the instrumental cue. 
In contrast to the context dependent over-imitation demonstrated by the 3-yearolds, with the exception of the 'silly' prompt the older children over-imitated at relatively high levels irrespective of whether the pre-demonstration instruction intimated either an instrumental, or a conventional, context. To some extent this result appeared somewhat counter intuitive as we may have expected the older children to show more flexibility and ability to capitalize on the information contained in the initial framing as a consequence of their better developed cognitive and social capabilities. However, this pattern of results is not without precedent as $\mathrm{Yu}$ and Kushnir (2014) also found an increase in over-imitation from 2 to 4 years following the initial establishment of different social contexts (copy game or instrumental game). Yu and Kushnir proposed that a possible explanation for this pattern of results is that children in the preschool period become increasingly influenced by the acquisition of social norms and conventions, drawing on pedagogical cues provided by the model (Csibra \& Gergely, 2009) in order to learn the normative way of using the object (Kenward et al., 2011; Kenward, 2012). This hypothesis is supported by the finding that children across the preschool period become increasingly sensitive to conventional activities in the domains of games (e.g., Rakoczy, Warneken, \& Tomasello, 2008; 2009), and artifact use (Casler, Terziyan, \& Greene, 2009); Wohlgelernter, Diesendruck \& Markson, 2010). Alternatively, it may be that the social affiliative element of imitation becomes increasingly important (Nielsen \& Blank, 2011), with the function of imitation (learning versus affiliation) changing at different developmental time-points (Uzgiris, 1981).

An alternative explanation is that rather than reflecting conventional and instrumental framing, the 'How to do it' versus 'One way to do it' task instructions may have suggested different levels of model competence with 'One way' suggesting uncertainty, or a lack of expertise. If the model was perceived as having a lack of expertise then the multiple irrelevant tool insertions may have appeared as mistaken attempts to retrieve the toy, 
resulting in the more selective copying by the younger children. However, this explanation would appear unlikely as previous over-imitation studies have shown that as children age they become increasingly, rather than decreasingly, selective in who they copy (McGuigan et al., 2011; Wood et al., 2012). In addition previous studies have shown that the inclusion of a pre-demonstration statement which directly indicated that the model was not an expert in the task ("This is a new game, I have never seen it before, I don't know how to do it") failed to reduce the occurrence of over-imitation (Wood et al., 2012), suggesting that it was unlikely that the current participants were responding on the basis of perceived model expertise. Whatever the precise source of selective over-imitation it appears that the relationship between the age and motivation of the observer, the context in which the task is presented, and the observer's relationship with the model is more complex than previously thought, and will require further exploration in future research.

That young children readily adopted the model's actions when the task was presented conventionally fits well with the recent finding that preschool children have been shown to conform to the behavior displayed by a group majority. Conformity, that is, adopting the most common behavior witnessed (Claidière \& Whiten, 2012), has been demonstrated across multiple domains, including the perceptual domain (Corriveau \& Harris, 2010; Haun \& Tomasello, 2011), the domain of verbal testimony (Chen, Corriveau, \& Harris, 2012), and the domain of object use (Haun, Rekers, \& Tomasello, 2012; McGuigan \& Robertson, 2015). Across these studies young children frequently adopted the approach of the majority, even in situations where the behavior of the group was clearly incorrect, or inefficient. Although it can on such occasions appear somewhat irrational, when viewed from the perspective of cultural evolution, conformity to majority behavior is likely to be an extremely adaptive strategy which can promote in-group homogeneity and between-group 
heterogeneity (Boyd \& Richerson, 2009; Haun, van Leeuwen, \& Edelson, 2013; Henrich \& Boyd, 1998).

Similarly, the high levels of over-imitation witnessed in the current study are likely reflective of this adaptive conformist tendency. However, despite the older children evidencing blanket conformity, our findings showed, somewhat unexpectedly, that very young children are not necessarily conformist, rather they responded flexibly depending upon how the task was presented. It may be that the pressure to conform increases during development, with the result that children lose their ability to dissent when presented with a cue to act counter to the conventional behavior. Why children become more extremely conformist is a question ripe for future research. Perhaps an early ability to engage in conformist behavior is replaced by a more powerful conformist tendency as a result of social experience in the form of increased exposure to cultural norms and conventions.

Alternatively it may be that cognitive changes occur during development which enable children to become more sensitive to social aspects of the social learning context including developments in theory of mind, and an increasing ability to read the intentionality of the model (McGuigan \& Graham, 2010). Future dissection of when, and how, this conformist tendency develops across childhood is crucial as the ability to adopt the conventional behavior displayed by our cultural group is likely to be a capacity which is fundamental to human culture as we know it.

\section{Acknowledgements}

This research was funded under the Undergraduate Research Internship Programme (URIP) organized by the University of St Andrews. We are also grateful to the Royal Zoological Society of Scotland (RZSS) for allowing us to collect data at the Budongo Trail Centre of the Edinburgh Zoo, UK. 


\section{References}

Boyd, R., \& Richerson, P. J. (2009). Culture and the evolution of human cooperation. Philosophical Transactions of the Royal Society B, 364, 3281-3288.

Brugger, A., Lariviere, L. A., Mumme, D. L., \& Bushnell, E. W. (2007). Doing the right thing: Infants' selection of actions to imitate from observed event sequences. Child Development, 78, 806-824.

Buchsbaum, D., Gopnik, A., Griffiths, T. L., \& Shafto, P. (2011). Children's imitation of causal action sequences is influenced by statistical and pedagogical evidence. Cognition, $120,331-340$.

Berl, R. E. W., \& Hewlett, B. S. (2015). Cultural Variation in the Use of Overimitation by the Aka and Ngandu of the Congo Basin. PLoS ONE, 10, e0120180.

Casler, K., Terziyan, T. \& Greene, K. (2009). Toddlers view artifact function normatively. Cognitive Development, 24, 240-247.

Chen, E. E., Corriveau, K. H., \& Harris, P. L. (2012). Children trust a consensus composed of outgroup members--but do not retain that trust. Child Development, 84, 269-282.

Claidière, N., \& Whiten, A. (2012). Integrating the study of conformity and culture in humans and nonhuman animals. Psychological Bulletin, 138, 126-145.

Clegg, J. M., and Legare, C. H. (in press) Instrumental and conventional interpretations of behavior are associated with distinct behavioral outcomes in early childhood. Child Development.

Corriveau, K. H., \& Harris, P. L. (2010). Preschoolers (sometimes) defer to the majority in making simple perceptual judgments. Developmental Psychology, 46, 437-445.

Csibra, G., \& Gergely, G. (2009). Natural pedagogy. Trends in Cognitive Sciences, 13, 148153. 
Haun, D. B. M., Rekers, Y., \& Tomasello, M. (2012). Majority-biased transmission in chimpanzees and human children, but not orangutans. Current Biology, 22, 727-731.

Haun, D. B. M., \& Tomasello, M. (2011). Conformity to peer pressure in preschool children. Child Development, 82, 1759-1767.

Haun, D. B. M., van Leeuwen, E. J. C., \& Edelson, M. G. (2013). Majority influence in children and other animals. Developmental Cognitive Neuroscience, 3, 61-71.

Henrich, J., \& Boyd, R. (1998). The Evolution of Conformist Transmission and the Emergence of Between-Group Differences. Evolution and Human Behavior, 19, $215-$ 241.

Herrmann, P. A., Legare, C. H., Harris, P. L., \& Whitehouse, H. (2013). Stick to the script: the effect of witnessing multiple actors on children's imitation. Cognition, 129, 536-543.

Hoehl, S., Zettersten, M., Schleihauf, H., Grätz, S., \& Pauen, S. (2014). The role of social interaction and pedagogical cues for eliciting and reducing overimitation in preschoolers. Journal of Experimental Child Psychology, 122, 122-133.

Hopper, L. M., Lambeth, S. P., Schapiro, S. J., \& Whiten, A. (2008). Observational learning in chimpanzees and children studied through "ghost" conditions. Proceedings of the Royal Society B, 275, 835-840.

Horner, V., \& Whiten, A. (2005). Causal knowledge and imitation/emulation switching in chimpanzees (Pan troglodytes) and children (Homo sapiens). Animal Cognition, 8, 164181.

Kenward, B. (2012). Over-imitating preschoolers believe unnecessary actions are normative and enforce their performance by a third party. Journal of Experimental Child Psychology, 112, 195-207. 
Kenward, B., Karlsson, M., \& Persson, J. (2011). Over-imitation is better explained by norm learning than by distorted causal learning. Proceedings of the Royal Society B, 278, 1239-1246.

Keupp, S., Behne, T., \& Rakoczy, H. (2013). Why do children overimitate? Normativity is crucial. Journal of Experimental Child Psychology, 116, 392-406.

Keupp, S., Behne, T., Zachow, J., Kasbohm, A., \& Rakoczy, H. (2015). Over-imitation is not automatic: Context-sensitivity in children's over-imitation and action interpretation of causally irrelevant actions. Journal of Experimental Child Psychology, 130, 163-175.

Legare, C. H., \& Nielsen, M. (in press). Imitation and innovation: The dual engines of cultural learning. Trends in Cognitive Sciences.

Legare, C. H., Wen, N. J., Herrmann, P.A., \& Whitehouse, H. (2015). Imitative flexibility and the development of cultural learning. Cognition, 142, 351-361.

Lyons, D. E., Damrosch, D. H., Lin, J. K., Macris, D. M., \& Keil, F. C. (2011). The scope and limits of overimitation in the transmission of artefact culture. Philosophical Transactions of the Royal Society B, 366, 1158-1167.

Lyons, D. E., Young, A. G., \& Keil, F. C. (2007). The hidden structure of overimitation. PNAS, 104, 19751-19756.

McGuigan, N. (2014). The influence of model status on the tendency of young children to over-imitate. Journal of Experimental Child Psychology, 116, 962-969.

McGuigan, N., Gladstone, D., \& Cook, L. (2012). Is the cultural transmission of irrelevant tool actions in adult humans (Homo Sapiens) best explained as the result of an evolved conformist bias? PLoSONE, 0050863

McGuigan, N., \& Graham, M. (2010). Cultural transmission of irrelevant tool actions in diffusion chains of 3- and 5-year-old children. European Journal of Developmental Psychology, 7, 561-577. 
McGuigan, N., Makinson, J., \& Whiten, A. (2011). From over-imitation to super-copying: adults imitate causally irrelevant aspects of tool use with higher fidelity than young children. British Journal of Psychology, 102, 1-18.

McGuigan, N. \& Robertson, S. (2015). The influence of peers on the tendency of 3- and 4year-old children to over-imitate. Journal of Experimental Child psychology, 36, 42-54.

McGuigan, N., Whiten, A., Flynn, E., \& Horner, V. (2007). Imitation of causally opaque versus causally transparent tool use by 3 - and 5-year-old children. Cognitive Development, 22, 353-364.

Nagell, K., Olguin, R. S., \& Tomasello, M. (1993). Processes of social learning in the tool use of chimpanzees (Pan troglodytes) and human children (Homo sapiens). Journal of Comparative Psychology, 107, 174-186.

Nielsen, M. (2006). Copying actions and copying outcomes: Social learning through the second year. Developmental Psychology, 42, 555-565.

Nielsen, M., \& Blank, C. (2011). Imitation in young children: When who gets copied is more important than what gets copied. Developmental Psychology, 47, 1050-1053.

Nielsen, M., Mushin, I., Tomaselli, K., \& Whiten, A. (2014). Where culture takes hold: 'Overimitation' and its flexible deployment in Western, Aboriginal and Bushmen children. Child Development, 85, 2169-2184.

Nielsen, M., Kapitány \& Elkins, R. (2015). The perpetuation of ritualistic actions as revealed by young children's transmission of normative behavior. Evolution and Human Behavior, 36, 191-198.

Nielsen, M., \& Tomaselli, K. (2010). Overimitation in Kalahari Bushman children and the origins of human cultural cognition. Psychological Science, 21, 729-736. 
Over, H., \& Carpenter, M. (2012). Putting the social into social learning: Explaining both selectivity and fidelity in children's copying behavior. Journal of Comparative Psychology, 126, 182-192.

Rakoczy, H., Brosche, N., Warneken, F., \& Tomasello, M. (2009). Young children's understanding of the context-relativity of normative rules in conventional games. British Journal of Developmental Psychology, 27, 445-456.

Rakoczy, H., Warneken, F., \& Tomasello, M. (2008). The sources of normativity: young children's awareness of the normative structure of games. Developmental Psychology, $44,875-881$.

Rakoczy, H., Warneken, F., \& Tomasello, M. (2009). Young children's selective learning of rule games from reliable and unreliable models. Cognitive Development, 24, 61-69.

Uzgiris, I. C. (1981). Two functions of imitation during infancy. International Journal of Behavioral Development, 4, 1-12.

Watson-Jones, R. E., Legare, C. H., Whitehouse, H., \& Clegg, J. M. (2014) Task-specific effects of ostracism on imitative fidelity in early childhood. Evolution and Human Behavior, 35, 204-210

Whiten, A., Custance, D. M., Gomez, J. C., Teixidor, P., \& Bard, K. A. (1996). Imitative learning of artificial fruit processing in children (Homo sapiens) and chimpanzees (Pan troglodytes). Journal of Comparative Psychology, 110, 3-14.

Whiten, A., \& Flynn, E. (2010). The transmission and evolution of experimental microcultures in groups of young children. Developmental Psychology, 46, 1694-1709.

Whiten, A., McGuigan, N., Marshall-Pescini, S., Hopper L. (2009). Emulation, imitation, overimitation and the scope of culture for child and chimpanzee. Philosophical Transactions of the Royal Society B, 364, 2117-2428. 
Wohlgelernter S., Gil Diesendruck \& Markson, L. (2010). What is a conventional object function? The effects of intentionality and consistency of use. Journal of Cognition and Development, 11, 269-292.

Wood, L. A., Kendal, R. L., \& Flynn, E. G. (2012). Context-dependent model-based biases in cultural transmission: children's imitation is affected by model age over model knowledge state. Evolution and Human Behavior, 33, 387-394.

Yu, Y., \& Kushnir, T. (2014). Social context effects in 2- and 4-year-olds' selective versus faithful imitation. Developmental Psychology, 50, 922-933.

FIGURES

Figure 1.

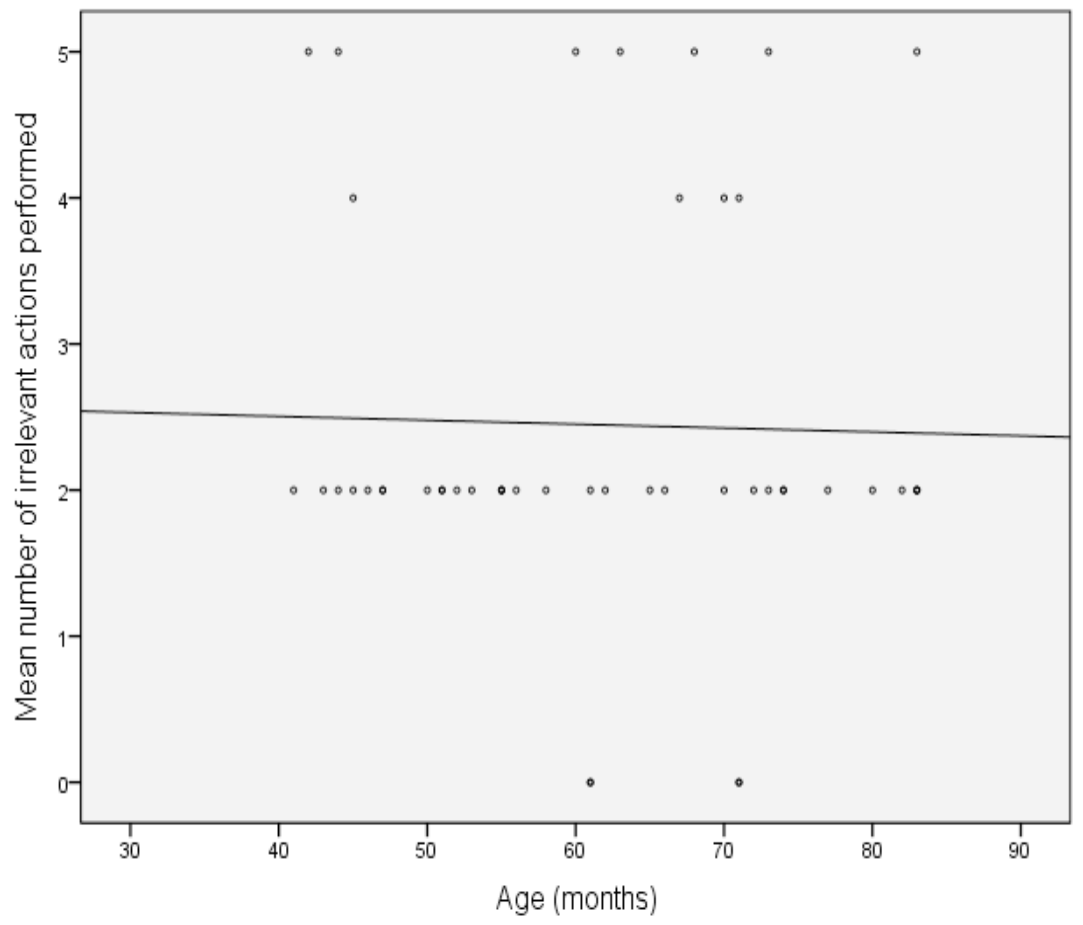




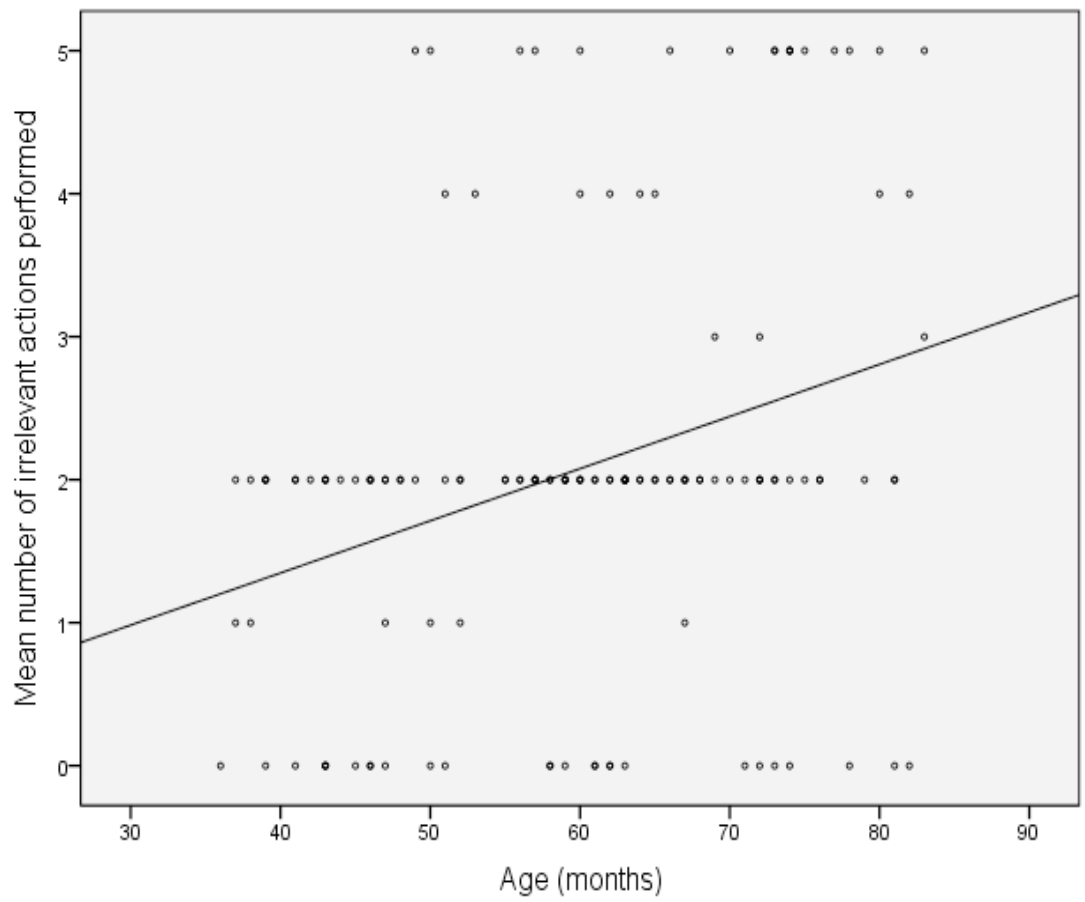

Figure 2.

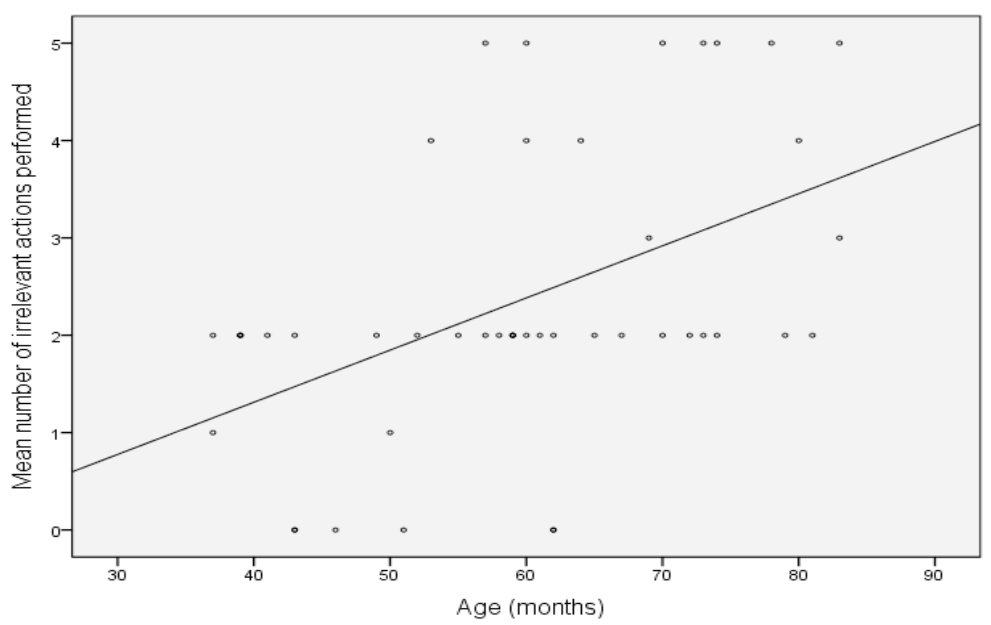



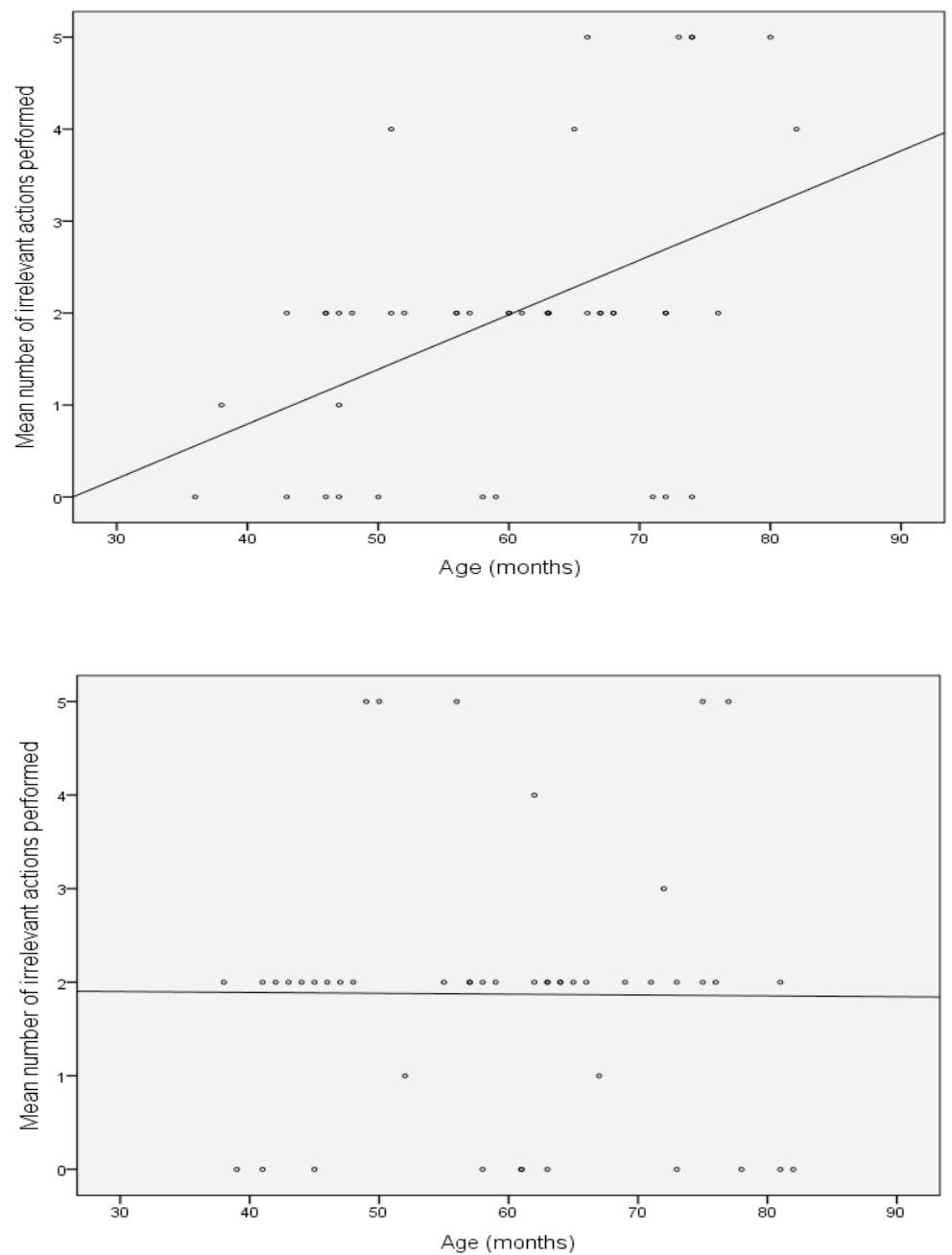

Figure Legends

Figure 1. Mean number of irrelevant actions performed as a function of age. Upper panel shows the mean number of irrelevant actions in the 'Single Solutions' condition. Lower panel shows the mean number of irrelevant actions in the three 'Multiple Solutions' conditions combined.

Figure 2. Mean number of irrelevant actions performed in each of the 'Multiple Solutions' conditions as a function of age. Upper panel shows the mean number of irrelevant actions in the 'Multiple Solutions-Your Turn' condition. Middle panel shows the mean number of irrelevant actions in the 'Multiple Solutions-However' condition. Lower panel shows the mean number of irrelevant actions in the 'Multiple Solutions-Silly' condition. 


\section{TABLE}

Table 1. Mean number of causally irrelevant actions and goal relevant actions performed in

\begin{tabular}{|c|c|c|c|c|c|c|c|c|c|c|c|c|c|c|c|c|}
\hline & \multicolumn{4}{|c|}{3 years } & \multicolumn{4}{|c|}{4 years } & \multicolumn{4}{|c|}{5 years } & \multicolumn{4}{|c|}{6 years } \\
\hline Condition & $\begin{array}{c}\text { SS } \\
(\mathrm{YT})\end{array}$ & $\begin{array}{c}\text { MS } \\
\text { (YT) }\end{array}$ & $\begin{array}{l}\text { MS } \\
(\mathrm{H})\end{array}$ & $\begin{array}{l}\text { MS } \\
\text { (S) }\end{array}$ & $\begin{array}{c}\mathrm{SS} \\
(\mathrm{YT})\end{array}$ & $\begin{array}{c}\mathrm{MS} \\
\text { (YT) }\end{array}$ & $\begin{array}{l}\text { MS } \\
(\mathrm{H})\end{array}$ & $\begin{array}{l}\text { MS } \\
\text { (S) }\end{array}$ & $\begin{array}{c}\mathrm{SS} \\
(\mathrm{YT})\end{array}$ & $\begin{array}{c}\text { MS } \\
\text { (YT) }\end{array}$ & $\begin{array}{l}\text { MS } \\
(\mathrm{H})\end{array}$ & $\begin{array}{l}\text { MS } \\
\text { (S) }\end{array}$ & $\begin{array}{c}\mathrm{SS} \\
(\mathrm{YT})\end{array}$ & $\begin{array}{c}\text { MS } \\
\text { (YT) }\end{array}$ & $\begin{array}{l}\text { MS } \\
(\mathrm{H})\end{array}$ & $\begin{array}{l}\text { MS } \\
\text { (S) }\end{array}$ \\
\hline Irrelevant & $\begin{array}{c}2.8 \\
(1.3)\end{array}$ & $\begin{array}{c}1.3 \\
(1.0)\end{array}$ & $\begin{array}{c}1.0 \\
(0.9)\end{array}$ & $\begin{array}{c}1.5 \\
(0.9)\end{array}$ & $\begin{array}{l}2.0 \\
(0)\end{array}$ & $\begin{array}{c}2.2 \\
(1.3)\end{array}$ & $\begin{array}{r}1.6 \\
(1.3)\end{array}$ & $\begin{array}{r}2.6 \\
(1.7)\end{array}$ & $\begin{array}{c}2.5 \\
(1.9)\end{array}$ & $\begin{array}{c}2.5 \\
(1.6)\end{array}$ & $\begin{array}{c}2.2 \\
(1.1)\end{array}$ & $\begin{array}{c}1.6 \\
(1.1)\end{array}$ & $\begin{array}{c}2.5 \\
(1.2)\end{array}$ & $\begin{array}{c}3.4 \\
(1.4)\end{array}$ & $\begin{array}{c}3.0 \\
(2.1)\end{array}$ & $\begin{array}{c}1.9 \\
(1.9)\end{array}$ \\
\hline Relevant & $\begin{array}{c}2.9 \\
(0.3)\end{array}$ & $\begin{array}{l}3.0 \\
(0)\end{array}$ & $\begin{array}{l}3.0 \\
(0)\end{array}$ & $\begin{array}{l}3.0 \\
(0)\end{array}$ & $\begin{array}{c}2.9 \\
(0.3)\end{array}$ & $\begin{array}{l}3.0 \\
(0)\end{array}$ & $\begin{array}{c}2.9 \\
(0.3)\end{array}$ & $\begin{array}{c}2.9 \\
(0.3)\end{array}$ & $\begin{array}{c}2.7 \\
(0.5)\end{array}$ & $\begin{array}{c}2.9 \\
(0.4)\end{array}$ & $\begin{array}{l}3.0 \\
(0)\end{array}$ & $\begin{array}{c}2.9 \\
(0.4)\end{array}$ & $\begin{array}{c}2.8 \\
(0.4)\end{array}$ & $\begin{array}{l}3.0 \\
(0)\end{array}$ & $\begin{array}{c}2.9 \\
(0.3)\end{array}$ & $\begin{array}{c}2.8 \\
(0.4)\end{array}$ \\
\hline Total & $\begin{array}{c}5.7 \\
(1.2)\end{array}$ & $\begin{array}{c}4.3 \\
(1.0)\end{array}$ & $\begin{array}{r}4.0 \\
(0.9)\end{array}$ & $\begin{array}{c}4.5 \\
(0.9)\end{array}$ & $\begin{array}{c}4.9 \\
(0.3)\end{array}$ & $\begin{array}{c}5.2 \\
(1.3)\end{array}$ & $\begin{array}{c}4.5 \\
(1.3)\end{array}$ & $\begin{array}{c}5.5 \\
(1.8)\end{array}$ & $\begin{array}{c}5.1 \\
(2.0)\end{array}$ & $\begin{array}{c}5.4 \\
(1.6)\end{array}$ & $\begin{array}{c}5.2 \\
(1.1)\end{array}$ & $\begin{array}{c}4.5 \\
(1.3)\end{array}$ & $\begin{array}{c}5.3 \\
(1.3)\end{array}$ & $\begin{array}{c}6.4 \\
(1.4)\end{array}$ & $\begin{array}{c}5.9 \\
(2.1)\end{array}$ & $\begin{array}{c}4.7 \\
(1.8)\end{array}$ \\
\hline
\end{tabular}

each age group of each condition.

Notes. 1. SS $(\mathrm{YT})=$ Single Solution-Your Turn; 2. MS(YT) = Multiple Solutions-Your Turn;

3. $\mathrm{MS}(\mathrm{H})=$ Multiple Solutions-However; 4. MS(S) = Multiple Solutions-Silly; 7. Max number of causally irrelevant actions $=5 ; 8$. Max number of goal relevant actions $=3 ; 9$. Max total $=8$. Standard deviations are shown in brackets. 